\title{
Integrin-independent support of cancer drug resistance by tetraspanin CD151
}

\author{
Soonyean Hwang ${ }^{1} \cdot$ Takayuki Takimoto $^{1,2} \cdot$ Martin E. Hemler $^{1}$ (])
}

Received: 5 October 2018 / Revised: 21 December 2018 / Accepted: 15 January 2019 / Published online: 18 February 2019

(c) The Author(s) 2019

\begin{abstract}
Tetraspanin protein CD151 has typically been studied as binding partner and functional regulator of laminin-binding integrins. However, we show here that CD151 supports anti-cancer drug resistance independent of integrins. CD151 ablation sensitized multiple tumor cell types to several anti-cancer drugs (e.g., gefitinib and camptothecin), thus increasing apoptosis, as seen using cleaved caspase-3, cleaved PARP (poly (ADP-ribose) polymerase), annexin V, and propidium iodide staining assays. Drug sensitization due to CD151 ablation is integrin-independent, because, (1) effects occurred in cells when integrins were unengaged with ligand, (2) integrin ablation ( $\alpha 3$ and $\alpha 6$ subunits) did not mimic effects of CD151 ablation, (3) the $\mathrm{CD} 151^{\mathrm{QRD}}$ mutant, with diminished integrin association, and $\mathrm{CD} 151^{\mathrm{WT}}$ (unmutated CD151) similarly reconstituted drug protection, and (4) treatment with anti-cancer drugs selectively upregulated intracellular nonintegrin-associated CD151 (NIA-CD151), consistent with its role in drug resistance. Together, these results suggest that upregulated CD151 expression may support not only typical integrin-dependent functions, but also integrin-independent survival of circulating (and possibly metastatic) cancer cells during anti-cancer drug therapy.
\end{abstract}

Keywords CD151 $\cdot$ Integrins $\cdot$ Drug resistance $\cdot$ Apoptosis

\section{Introduction}

CD151, a $30 \mathrm{kD}$ tetraspanin protein family member, is widely expressed in many tissues and cancer cell types [1-3]. CD151 expression is increased in breast, prostate, lung, colon, skin, and other cancers, and elevated CD151 expression correlates with advanced stage and poor prognosis in many of these human cancers [3-5]. CD151 may

Soonyean Hwang and Takayuki Takimoto contributed equally to this work.

Electronic supplementary material The online version of this article (https://doi.org/10.1007/s00018-019-03014-7) contains supplementary material, which is available to authorized users.

Martin E. Hemler

martin_hemler@dfci.harvard.edu

1 Department of Cancer Immunology and Virology, Rm SM-520C, Dana-Farber Cancer Institute, 450 Brookline Ave, Boston, MA 02215, USA

2 Present Address: Department of Internal Medicine, National Hospital Organization Kinki-Chuo Chest Medical Center 1180 Nagasone-cho, Kita-ku, Sakai, Osaka 591-8555, Japan variably support tumor initiation, promotion, progression, metastasis, angiogenesis, and drug resistance [6-10]. To date, CD151 has mostly been studied as a closely associated cell surface partner and regulator of laminin-binding integrins (e.g., $\alpha 3 \beta 1, \alpha 6 \beta 1, \alpha 6 \beta 4$, and $\alpha 7 \beta 1$ ) [11-13]. Genetic evidence reinforces the close functional connection between CD151 [14-16] and laminin-binding integrins $[17,18]$. However, CD151 also can appear as a nonintegrinassociated molecule, selectively recognized by anti-CD151 $\mathrm{mAb}$ that bind to a CD151 epitope(s) only exposed when it is not bound to integrin [19-22]. A correlation between nonintegrin-associated CD151 (NIA-CD151) and diminished prostate cancer patient survival [21] underscores the potential importance of NIA-CD151.

The absence of CD151 sensitized normal mouse skin cells to trastuzumab, camptothecin, DMBA (7,12-dimethylbenz[ $\alpha]$ anthracene), and agents targeting Jak2/Tyk2 and STAT3 [6]. These findings hinted at a possible link between CD151 and general drug resistance. Cancer drug resistance, intrinsic or acquired, can arise due to a variety of mechanisms, including tumor cell heterogeneity, drug efflux and metabolism, and drug-induced genetic or epigenetic cellular alterations [23]. Because integrin-mediated 
cell adhesion contributes to drug resistance [24], it was expected that potential CD151 contributions to drug resistance would also involve integrins. Indeed, in the special case of drugs targeting ErbB2 and focal adhesion kinase (FAK), CD151 supported drug resistance by a mechanism involving laminin and laminin-binding integrins [25]. However, in sharp contrast to those previous integrin-dependent results [25], we here show that nonintegrin-associated CD151 (NIA-CD151) facilitates a more general and previously unreported anti-cancer drug-induced apoptosis.

\section{Materials and methods}

\section{Cells, antibodies, and other reagents}

A431 (epidermoid carcinoma), MDA-MB-231 (breast carcinoma), and A549 (lung carcinoma) cells were from the American Type Culture Collection (ATCC, Manassas, VA, USA) and maintained in Dulbecco's modified Eagle's medium with $10 \%$ fetal calf serum (Sigma, MO, USA), and $1 \%$ penicillin-streptomycin (Invitrogen, Grand Island, NY, USA) at $37^{\circ} \mathrm{C}$ in humidified $5 \% \mathrm{CO}_{2}$. Monoclonal antibodies to CD151 were 11B1 (gift from B. Copeland), TS151r (gift from E. Rubinstein), 5C11, 1A5 (gift from A. Zijlstra), and 14A2H1 (BD Biosciences, San Jose, CA, USA). TS151r and 1A5 selectively recognize the nonintegrin-bound form of CD151 (NIA-CD151) [21, 22]. Antibodies to cleaved caspase-3, PARP, p-mTOR/mTOR, p-AKT/AKT, and p-ERK/ERK were purchased from Cell Signaling Technologies (Danvers, MA, USA). Antibodies to CD9 (MM2/57), integrin $\beta 4$, glyceraldehyde-3-phosphate dehydrogenase (GAPDH), and $\beta$-actin were from EMD Millipore (Billerica, MA, USA). Antibodies to integrins $\alpha 6, \alpha 3$, and CD81 and normal mouse IgG were from Santa Cruz Biotechnology (Santa Cruz, CA, USA). Antibodies to CD49f ( $\alpha 6$ integrin), phospho-EGFR, and EGFR were from BD Biosciences (Franklin Lakes, NJ, USA). Gefitinib was from Cayman Chemical (Ann Arbor, MI, USA) and DMSO, camptothecin, cisplatin, and phorbol 12-myristate 13-acetate (PMA), were from Sigma-Aldrich. Nifuroxazide was from Dr. David Frank, Dana-Farber Cancer Institute. Lapatinib and U0126 were from LC Laboratories (Woburn, MA, USA). RNAi: siGENOME human CD151 siRNA (D-003637-04), siGENOME human CD9 siRNA (D-017252-04), and siGENOME human CD81 siRNA (D-017257-05) were from Dharmacon, Inc. (Chicago, IL, USA).

\section{Cell culture and drug treatment}

All cells were routinely cultured in $10 \%$ fetal calf serum, while adherent to tissue culture plastic. Drug treatment (at times and doses indicated in legends) was typically carried out for cells adherent to tissue culture plastic, in the presence of $10 \%$ serum. Exceptions are as follows: in Fig. 2c-e, A431 cells (labeled as "Nonadherent") were treated with drugs while plated on polyHEMA (which prevented cell adhesion). In Supplemental Fig. 2a, A431 cells (labeled as detached) were drug-treated while suspended in a conical plastic tube, with occasional mild agitation.

\section{CRISPR/Cas9-mediated gene deletion and reconstitution}

Lentiviral particles containing CD151 gRNA and Cas9 were produced as described previously [26]. CD151 gRNA-1 (5'CACCGGTAAACAGCAGGTACTTG-3'), gRNA-2 (5'CACCGGCAGTGGGCATCTGGACGC-3'), and gRNA-3 (5'-CACCGGTGGCCAGGTAGGTGCCTG-3') (IDT DBA, Coralville, USA). Human nonspecific control gRNA [27] was a gift from TC Cheong. CD $151^{\mathrm{WT}}$ and CD $151^{\mathrm{QRD}}$ mutant template plasmids [13] were gifts from Dr. C.S. Stipp. Silent mutations were introduced into CD $151^{\text {WT }}$ and CD $151^{\text {QRD }}$ to remove protospacer adjacent motif (PAM) sites and cloned into pLenti CMV Blast empty (w263-1) (Addgene, Cambridge, MA, USA).

\section{Immunoprecipitation and immunoblotting}

Cell lines were lysed in $50 \mathrm{mM}$ HEPES, $150 \mathrm{mM} \mathrm{NaCl}$, $5 \mathrm{mM} \mathrm{MgCl}_{2}, 1 \%$ TritonX-100 (1\% Brij 97, or 1\% Brij 99), $0.5 \mathrm{mM}$ PMSF and protease inhibitor cocktail (Roche Diagnostics, Indianapolis, IN, USA). After $60 \mathrm{~min}$ shaking at $4{ }^{\circ} \mathrm{C}$, lysates were collected and centrifuged $(14,000 \times \mathrm{g}$, $10 \mathrm{~min}, 4^{\circ} \mathrm{C}$ ), and then, immunoprecipitation was carried out prior to immunoblotting. Densitometry quantitation of protein after blotting was performed using Image Quant, version 5.2 software (GE Healthcare, Waukesha, WI, USA) or ImageJ 1.x (National Institutes of Health, Bethesda, MD). Co-immunoprecipitation of CD151 and PKC $\alpha$ was performed as described [28].

\section{RNA isolation and semi-quantitative real-time PCR}

mRNA was isolated from MDA-MB-231 and A431 cells and amplified using OneStep RT-PCR Kit (Qiagen, Venlo, Netherlands). Human CD151 mRNA primers: Fwd 5'-ACT TCATCCTGCTCCTCATCAT-3', Rev 5'-TCCGTGTTC AGCTGCTGGTA-3'. Human GAPDH mRNA primers: Fwd 5'-GGCATCCTGGGCTACACTGA-3', Rev 5'-GTG GTCGTTGAGGGCAATG-3'. 


\section{Immunofluorescence and phase contrast microscopy}

For confocal analyses, cells on coverslips were fixed with $4 \%$ paraformaldehyde at $4{ }^{\circ} \mathrm{C}$ for $10 \mathrm{~min}$, permeabilized by $0.1 \%$ Triton X-100 in PBS for 5 min, blocked with 2\% BSA in PBS (w/v) for $1 \mathrm{~h}$, and immunolabeled with primary, followed by secondary antibody (Alexa 488 or Alexa 594-conjugated IgG) alone or combined (Invitrogen, Carlsbad, CA, USA). Coverslips were mounted with ProLong Gold antifade mounting media containing DAPI. Cells were visualized using Yokogawa spinning disk confocal microscope (Yokogawa, Japan). Phase contrast images were obtained with Nikon Eclipse TE300 inverted microscope analyzed with Spot software (SPOT Imaging Sterling Heights, MI, USA).

\section{Cell death assay}

Apoptosis was determined using cleaved caspase-3 (immunoblotting), Cell Death Detection ELISA kit (Roche Molecular Biochemicals, Mannheim, Germany), and FITC Annexin V Apoptosis Detection Kit 1 (BD Pharmingen, Billerica, MA, USA).

\section{Flow cytometry}

Adherent cells were detached (using trypsin), and then, cell surface expression of CD151 was quantitated by flow cytometry (FACS Calibur, Becton-Dickinson, Bedford, MA, USA, with processing by FlowJo software, Ashland, OR, USA). Annexin $\mathrm{V}$ and propidium iodide staining were quantitated by flow cytometry (FACSAria II, with FACSDiva software analysis, BD Biosciences, Franklin Lakes, NJ, USA) with a minimum of 20,000 cells analyzed in each experiment.

\section{Statistics}

Statistical significance was calculated using two-tailed unpaired Student's $t$ test (Figs. 1b, d, 2b, d, 3e).

\section{Results}

\section{CD151 attenuates anti-cancer drug-induced apoptosis}

CD151 ablation experiments indicate that CD151 protects cancer cells from anti-cancer drugs. Treatment of cancer cell
A
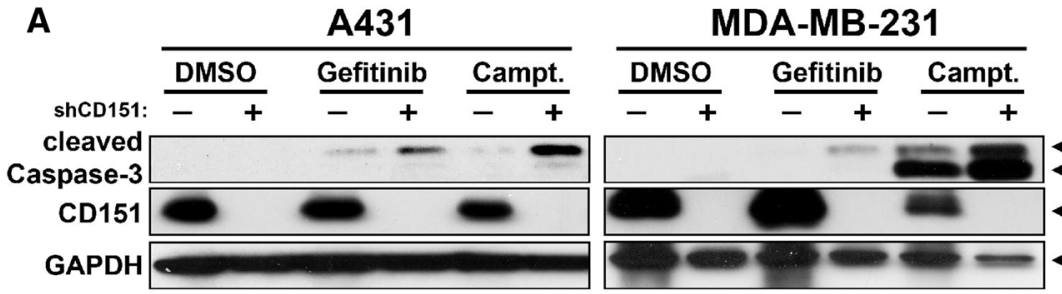

MDA-MB-231

C

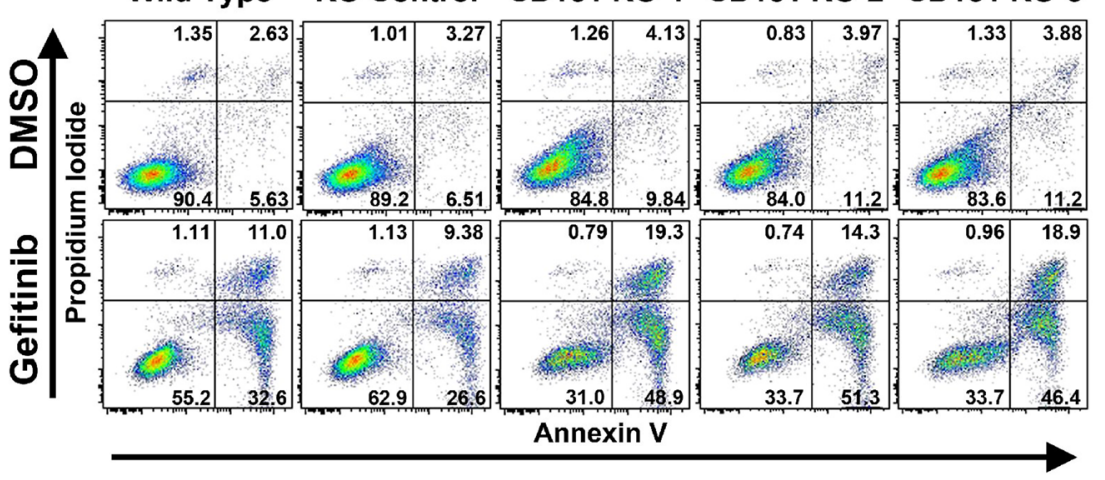

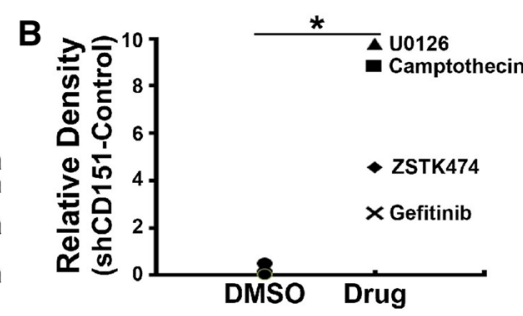

Fig. 1 CD151 ablation increases drug-induced apoptosis. a A431 and MDA-MB-231 cells \pm shRNA-mediated CD151 knockdown were treated for with gefitinib $(20 \mu \mathrm{M}, 24 \mathrm{~h})$ or camptothecin $(1 \mu \mathrm{M}$, $48 \mathrm{~h})$ or DMSO (1:1000, vehicle control). Cell lysates were blotted for cleaved caspase-3, CD151 or GAPDH as indicated. b A431 cells, \pm CD151 knockdown, were treated with DMSO (1:1000), Gefitinib $(10 \mu \mathrm{M})$, Camptothecin $(1 \mu \mathrm{M})$, ZSTK474 $(1 \mu \mathrm{M})$, and U0126 $(10 \mu \mathrm{M})$ for $6 \mathrm{~h}$, cell lysates were blotted for cleaved caspase-3, and results were quantitated. ${ }^{*} p<0.05$ (comparing four drug treatments with three DMSO treatments). c A431 cells with or without CRISPR/ Cas9 CD151 deletion were treated with DMSO or gefitinib $(5 \mu \mathrm{M}$ for $48 \mathrm{~h}$ ) and then dually stained with annexin $\mathrm{V}$ and propidium iodide. $\mathbf{d}$ A431 cells were treated with gefitinib, 5-fluorouracil, or camptothecin for $48 \mathrm{~h}$. Bars represent ratios of cells positive for annexin $\mathrm{V}$ and/or propidium iodide divided by double negative cells (lower left quadrants in panel c). ${ }^{*} p<0.05$ (comparing three CD151 knockout cell lines with and wild-type and gRNA control cell lines) 
A

A431 Adherent Cells

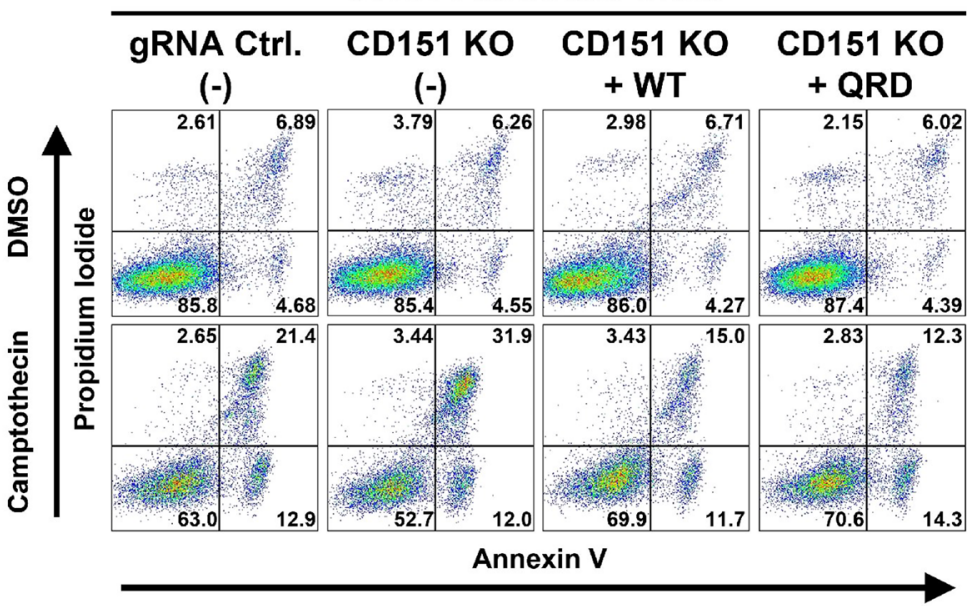

C

A431 Nonadherent Cells

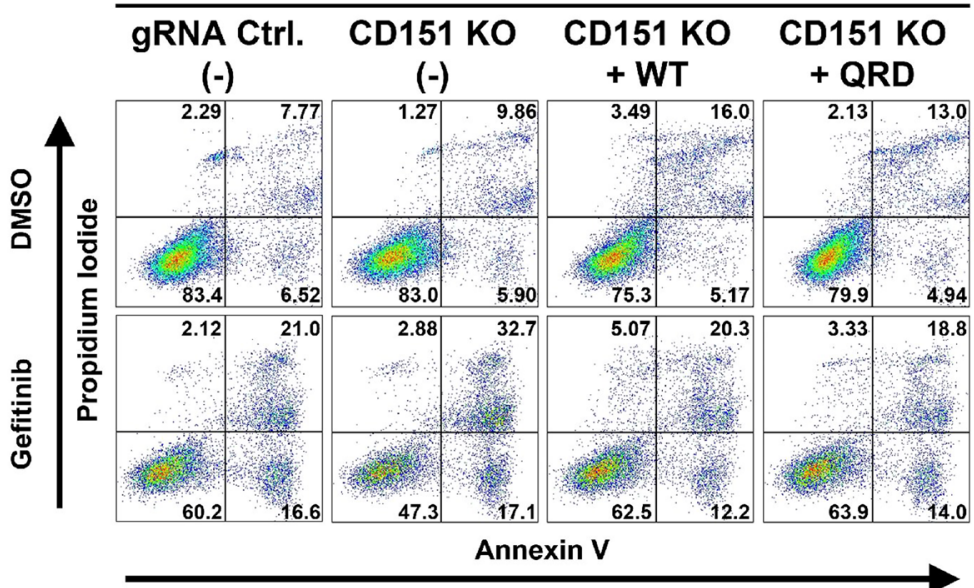

E

A431 Nonadherent Cells

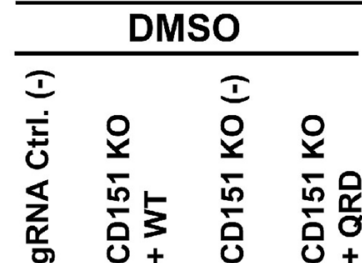

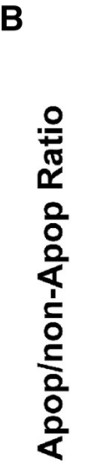

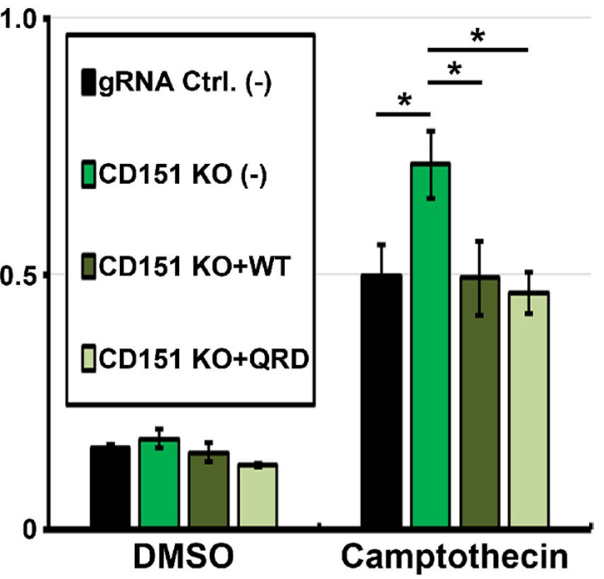

D

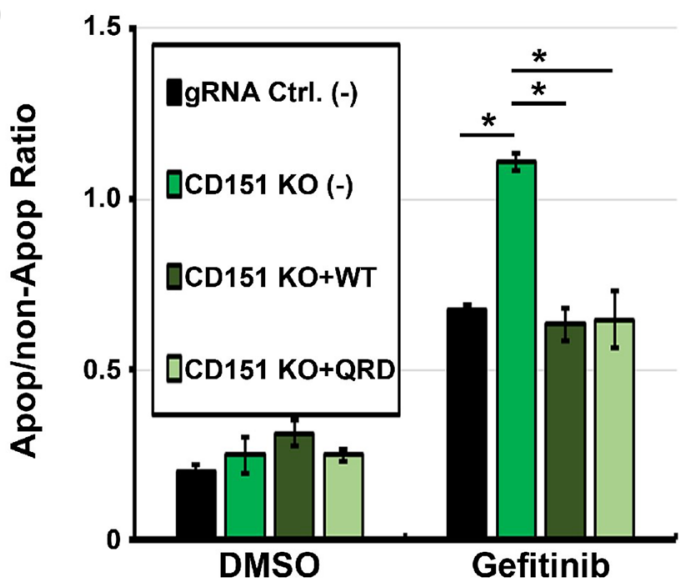

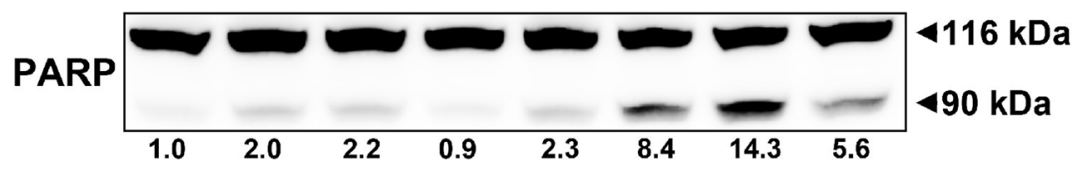

GAPDH

\section{$437 \mathrm{kDa}$}

Fig. 2 Rescue of anti-cancer drug-induced apoptosis in CD151 deleted cells. a Representative FACS density plots of annexin V and PI staining of adherent cell lines (CD151 knockout, CD151 ${ }^{\mathrm{WT}}$ and $\mathrm{CD} 151^{\mathrm{QRD}}$ reconstituted) treated with camptothecin $(50 \mathrm{nM}$ for $48 \mathrm{~h})$ in adherent conditions. b Quantitation of FACS results $(n=3$; $* p<0.05)$. c Representative FACS density plots of annexin V and PI staining of nonadherent cell lines (CD151 knockout, CD151 ${ }^{\mathrm{WT}}$ and
$\mathrm{CD} 151^{\mathrm{QRD}}$ reconstituted) treated with gefitinib (5 $\mu \mathrm{M}$ for $\left.48 \mathrm{~h}\right)$ on polyHEMA-coated surfaces. d Quantitation of FACS analysis $(n=3$; $* p<0.05$ ). e Immunoblot of apoptotic marker cleaved PARP in nonadherent cells treated with Camptothecin on polyHEMA-treated surfaces. Numbers indicate relative density of cleaved PARP normalized to that in DMSO-treated control cells 


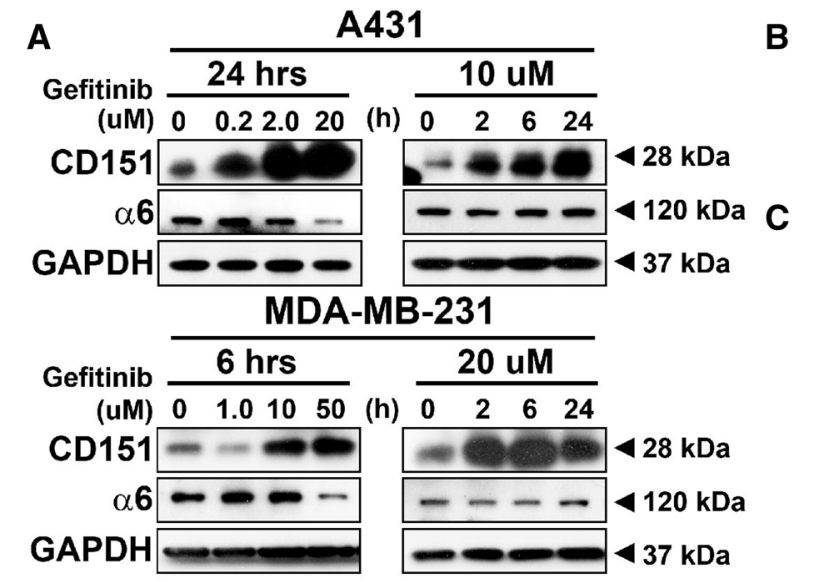

D A431
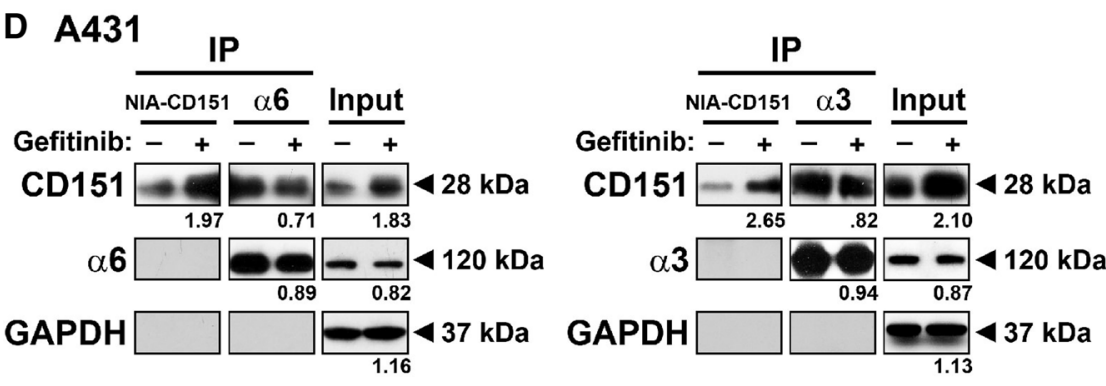

A431 DMso Gef. Lapa. Cis. Oxa. Camp. 5FU

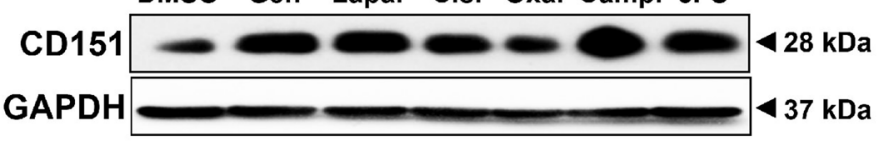

A431

Gefitinib

(h) \begin{tabular}{llll}
\hline & 2 & 6 & 24
\end{tabular}

CD151 $--628 \mathrm{kDa}$

$\alpha 3----4120 \mathrm{kDa}$

$\alpha 6---1120 \mathrm{kDa}$

$\beta 1--\infty<110 \mathrm{kDa}$

$\beta 4=0-210 \mathrm{kDa}$

$437 \mathrm{kDa}$

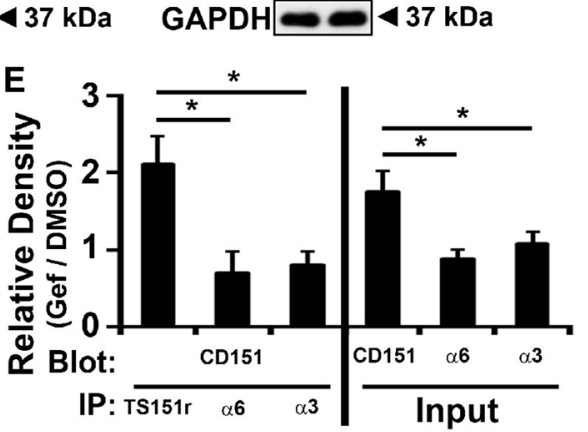

Fig. 3 Anti-cancer drug treatment increases nonintegrin-associated CD151 protein levels. a A431 and MDA-MB-231 cells were treated with gefitinib for indicated doses and times. Cell lysates were then blotted for CD151, integrin $\alpha 6$ subunit, and GAPDH. b A431 cells were treated with the indicated anti-cancer drugs gefitinib $(5 \mathrm{uM})$, lapatinib $(5 \mu \mathrm{M})$, cisplatin $(20 \mu \mathrm{M})$, oxaliplatine $(10 \mu \mathrm{M})$, camptothecin $(1 \mu \mathrm{M})$, and 5-fluorouracil $(10 \mu \mathrm{M})$ for $25 \mathrm{~h}$, and then, lysates were blotted for CD151 and GAPDH. c A431 (for indicated times) and A549 (24 h) cells were treated with gefitinib $(20 \mu \mathrm{M})$, and then, lysates were blotted for the indicated laminin-binding integrin subunits and GAPDH. D. After treatment with DMSO (1:1000) or gefi-

lines (A431 epidermoid carcinoma, MDA-MB-231 breast carcinoma) with gefitinib (EGFR inhibitor) or camptothecin (topoisomerase inhibitor) triggered apoptosis, as evidenced by elevated levels of cleaved caspase-3. The apoptotic response was markedly increased in shRNA-mediated CD151 knockdown cell lines (Fig. 1a; compare lanes 4, 6 with 3, 5 in top panels). A panel of anti-cancer drugs similarly yielded increased apoptosis (i.e., cleaved caspase-3) in CD151 knockdown cells compared to control shRNA cells (Fig. 1b). Consistent with increased apoptosis, gefitinibtreated CD151 knockdown cells also showed a significant decrease in cell growth (not shown). To complement our shRNA knockdown approach, CRISPR/Cas9-mediated deletion of CD151 was also carried out using three distinct gRNA's (Supplemental Fig. 1a, b). Consistent with CD151 knockdown results, CD151 gene deleted cells (CD151$\mathrm{KO}$ ) again displayed a marked increase in gefitinib-induced apoptosis, this time as seen by increased Annexin V staining (Fig. 1c; see cell percentage numbers in right panels). tinib $(20 \mu \mathrm{M})$ for $24 \mathrm{~h}, \mathrm{~A} 431$ cells were lysed in $1 \%$ Triton X-100. NIA-CD151 (mAb TS151r) and integrins $\alpha 3$ and $\alpha 6$ were immunoprecipitated. Recovered CD151, integrin $\alpha 6$, integrin $\alpha 3$, and GAPDH proteins from immunoprecipitation (lanes 1-4, 7-10) or from input lysate (lanes 5, 6, 11, and 12) were detected by blotting. As expected, anti-NIA-CD151 mAb TS151r did not co-immunoprecipitate integrin subunits (middle panels, lanes 1, 2, 7, 8). Numbers=gefitinib/DMSO treatment ratios from densitometry quantitation. e Quantitated ratios are shown for multiple experiments $($ mean $\pm \mathrm{SD} ; n=4 ; * p<0.01)$

In addition, treatment with multiple doses of gefitinib or chemotherapeutic compounds (5-fluorouracil, camptothecin) again significantly increased apoptosis in CD151 deleted cells (Fig. 1d). Consistent with results in Fig. 1a, CD151$\mathrm{KO}$ cells also showed increased drug-induced apoptosis as assessed by blotting for cleaved caspase-3 (not shown).

\section{CD151 drug protection effects are independent of laminin-binding integrins}

CD151 is a regulator of laminin-binding integrins, thus affecting cell motility, morphology, adhesion strengthening, and other functions [3, 19, 29]. However, effects of CD151 ablation on enhanced drug sensitivity remained obvious even when nonadherent cells (i.e., with integrins not engaging ligand) were treated with gefitinib. In fact, enhanced sensitivity due to CD151 ablation was even greater than that seen for adherent cells (Supplemental Fig. 2a). Furthermore, siRNA-mediated knockdown of 
integrin $\alpha 3$ and $\alpha 6$ subunits (major CD151 binding partners) did not result in increased gefitinib-induced apoptosis (Supplemental Fig. 2b). Together, these results suggest that the effects of CD151 abrogation on anti-cancer drug-induced apoptosis may be independent of CD151 association with integrins.

To further test whether CD151 association with laminin-binding integrins affects the regulation of anticancer drug-induced apoptosis, wild-type (CD151 ${ }^{\mathrm{WT}}$ ), and CD151 nonintegrin-binding QRD mutant (CD151 ${ }^{\mathrm{QRD}}$ ) were reconstituted into CD151 deleted cells (Supplemental Fig. 3a). Consistent with the published results [19], co-immunoprecipitation of CD151 ${ }^{\mathrm{QRD}}$, compared to $\mathrm{CD} 151^{\mathrm{WT}}$, shows markedly reduced association with $\alpha 3$ and $\alpha 6$ integrins (Supplemental Fig. 3b). However, despite differences in integrin association, both CD151 ${ }^{\text {WT }}$ and $\mathrm{CD} 151^{\mathrm{QRD}}$ were similarly able to restore resistance to camptothecin in $C D 151$ deleted adherent A431 cells (Fig. 2a, b) and resistance to gefitinib in CD151 deleted nonadherent A431 cells (Fig. 2c, d). Ability of both $\mathrm{CD} 151^{\mathrm{WT}}$ and $\mathrm{CD} 151^{\mathrm{QRD}}$ to restore protection against anti-cancer drug-induced cell death was further verified by diminished appearance of apoptotic marker cleaved PARP (Fig. 2e, lanes 6,8) in camptothecin treated nonadherent A431 cells. Morphology of A431 cells, grown at either low density or high density, was not noticeably altered due to the deletion of $C D 151$ or reconstitution with CD151 ${ }^{\text {WT }}$ or CD151 ${ }^{\mathrm{QRD}}$ (Supplemental Fig. 3c).

\section{Anti-cancer drug treatment increases levels of nonintegrin-associated CD151}

In various cancer cell lines (A431, MDA-MB-231, and A549 lung carcinoma) treated with gefitinib, we observed an increase in total CD151 protein levels in a dose- and timedependent manner (Figs. 3a, c, Supplemental Fig. 4a). An increase in CD151 was similarly observed in A431 cells in response to a panel of additional anti-cancer drugs (Fig. 3b). Notably, this increase in CD151 was not accompanied by consistent increases in integrins $(\alpha 3 \beta 1, \alpha 6 \beta 1$, and $\alpha 6 \beta 4)$ that typically associate with CD151 (Figs. 3a, c, Supplemental Fig. 4a). To test whether anti-cancer drugs selectively induced increases in integrin-associated or nonintegrin-associated CD151, immunoprecipitation experiments were performed. Upon gefitinib treatment, levels of nonintegrin-associated CD151 (NIA-CD151; selectively immunoprecipitated using mAb TS151r) were increased by 1.97 -fold (Fig. 3d, left panel) or by 2.65 -fold (right panel). By contrast, CD151 co-immunoprecipitated with integrin $\alpha 3$ (right panel) or $\alpha 6$ subunits (left panel) did not increase. A summary of results from multiple experiments confirmed that NIA-CD151 increased by $>$ twofold, whereas integrin-associated CD151

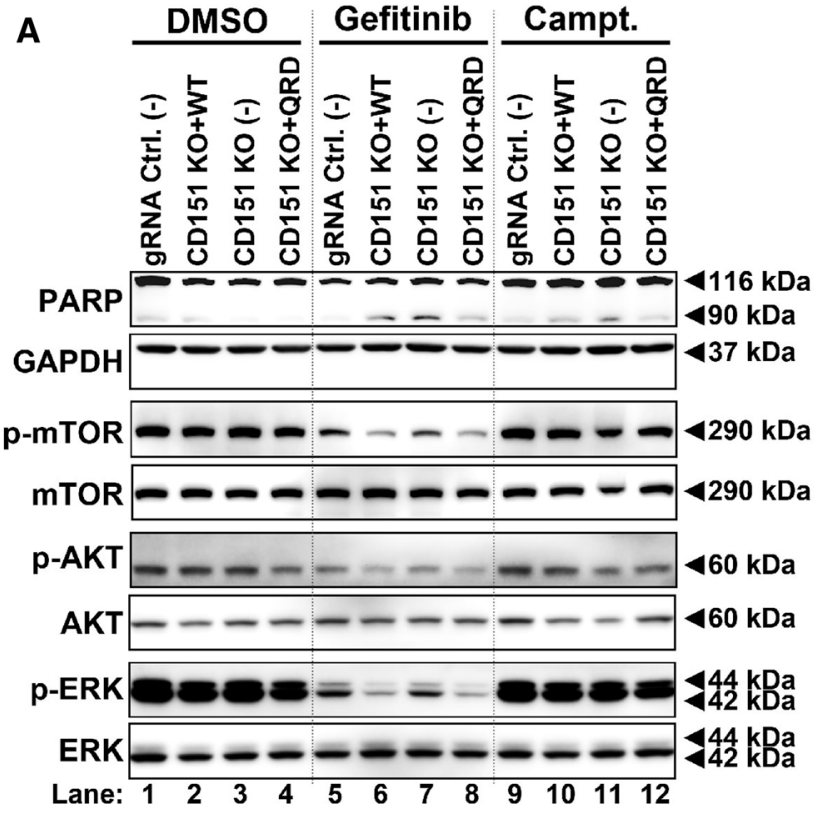

Fig. 4 Effects of CD151 changes on pro-survival signaling pathways. a A431 cells with $C D 151$ deleted and/or reconstituted, growing on polyHEMA-coated surfaces, were treated with DMSO, Gefitinib $(10 \mu \mathrm{M}, 6 \mathrm{~h})$ or camptothecin $(1 \mu \mathrm{M}, 6 \mathrm{~h})$. Cell lysates were then immunoblotted for phospho- and total mTOR, AKTh and ERK, as well as apoptosis marker cleaved PARP and GAPDH. b Quantitation relative protein levels are shown for cleaved PARP (left axis),
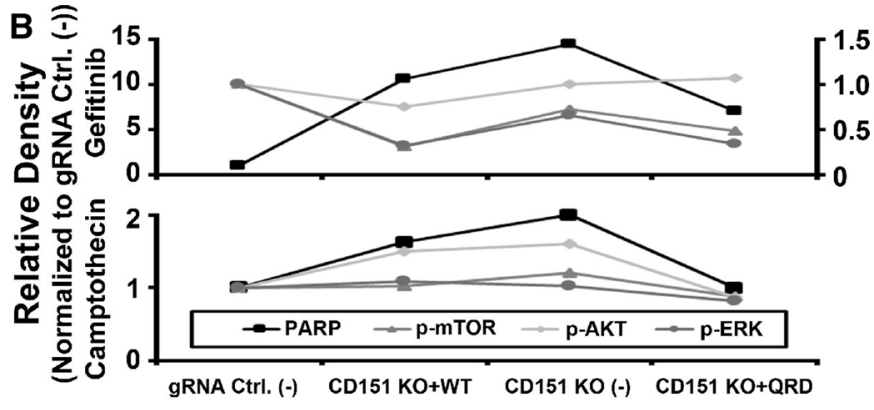

C

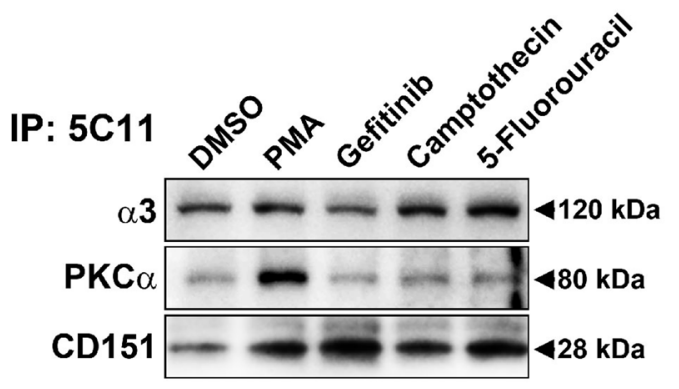

p-mTOR (right axis), p-AKT (right axis), and p-ERK (right axis) normalized to control cell line. c A431 cells were treated with control PMA, gefitinib $(20 \mu \mathrm{M})$, camptothecin $(1 \mu \mathrm{M})$, and 5-fluorouracil $(10 \mu \mathrm{M})$ for $8 \mathrm{~h}$. CD151 was immunoprecipitated (using mAb 5C11), and then, levels of recovered CD151 and co-immunoprecipitated $\mathrm{PKC} \alpha$ and integrin $\alpha 3$ were assessed by immunoblotting 
did not increase (Fig. 3e, left). Consistent with the results in Fig. 3a, b, total input CD151 was increased in Fig. 3d (by 1.83- and 2.10-fold) and in Fig. 3e (by $\sim 1.8$-fold), but not quite to the same extent as the increase in NIA-CD151. In another experiment (Supplemental Fig. 4b), A431 cells either expressed endogenous CD151 (WT) or were deleted for $C D 151$ and reconstituted with either flag-tagged WT CD151 (WT-F) or flag-tagged QRD CD151 (QRD-F), each under the regulation of a nonendogenous CMV promoter. Again, gefitinib treatment increased CD151 levels, as seen by immunoblotting using three different antibodies (Supplemental Fig. 4b). Increased CD151 was most obvious for mAb 1 A5 (recognizing NIA-CD151; top panel) and less obvious for antibodies (mAb 11B1 and anti-FLAG) that recognize total CD151 (next two panels). CD151 protein levels increased in response to gefitinib treatment Supplemental Fig. 4c, but in the same experiments, CD151 mRNA levels did not increase (Supplemental Fig. 4d). These results indicate that the drug-induced increase in CD151 protein does not involve endogenous promotors or enhancers, and does not require integrin association. In drug-treated cells, subcellular distribution of NIA-CD151 (detected using mAb TS151r) showed minimal overlap with integrin $\alpha 6$ (Supplemental Fig. 4e) or integrin $\alpha 3$ (not shown), consistent with NIA-CD151 having a role independent from integrins.

Although levels of total CD151 and NIA-CD151 were markedly increased upon gefitinib treatment (Supplemental Fig. $4 \mathrm{a}-\mathrm{c})$, these increases were not seen at the cell surface on any of the cell lines (A431, MDA-MB-231, and A549) when analyzed for either total CD151 (mAb 11B1 and 5C11) or NIA-CD151 (mAb TS151r) (Supplemental Fig. 5). In contrast, levels of intracellular NIA-CD151 (detected using mAb TS151r) were markedly increased in MDA-MB-231 cells treated with gefitinib (Supplemental Fig. 5b). Together, these results point to selective upregulation of intracellular NIA-CD151 in response to anti-cancer agents.

\section{CD151 acts as an inhibitor of apoptosis in response to anti-cancer drugs}

CD151 itself has not been shown to act as a signaling protein. However, it is implicated in regulating spatial and temporal recruitment of signaling proteins such as conventional PKCs and PI4K [28, 30], which can support pro-survival signaling pathways of cancer cells $[31,32]$. Hence, we expected that effects of CD151 deletion and CD151 reconstitution on apoptosis might be opposite to effects on survival, as assessed by phosphorylation of key pro-survival pathway mediators (AKT, ERK, and mTOR). However, no inverse correlation was observed. As indicated in Fig. 4a, the apoptotic marker cleaved PARP was substantially elevated in drug-treated cells lacking CD151 (lanes 7, 11), compared to drug-treated control cells (lanes 5, 9). However, AKT,
ERK and mTOR phosphorylations were not correspondingly decreased (lanes 7, 11 compared to 5, 9). Furthermore, in CD151 reconstituted cells (lanes 6, 8, 10, 12), levels of the apoptotic marker were partially diminished (compared to lanes 7, 11), but AKT, ERK, and mTOR phosphorylations were not correspondingly increased. Figure $4 \mathrm{~b}$ shows densitometric quantitation of results in Fig. 4a.

Induced association of $\mathrm{CPKC}$ with CD151 appears to play a key role in cellular growth/survival responses to agents such as EGF and the phorbol ester PMA [6]. However, association of PKC $\alpha$ with CD151 was not altered in cells treated with anti-cancer drugs compared to DMSO (Fig. 4c). In a positive control experiment, PMA did induce an increase in CD151-PKC $\alpha$ association (Fig. 4c, lane 2).

\section{Discussion}

Targeted ablation of tetraspanin protein CD151 sensitized tumor cells to a variety of anti-cancer drugs. Results were seen in multiple tumor cell lines, in which CD151 was either knocked down (using shRNA/RNAi) or knocked out (for the first time using CRISPR/Cas9). Increased apoptosis was indicated by increased levels of cleaved caspase-3, cleaved PARP, annexin V staining, and propidium iodide staining. The increased apoptotic response was associated with reduced growth of CD151-ablated, drug-treated cancer cells.

The previous studies of CD151 have emphasized its close physical association with laminin-binding integrins, and its role in regulating integrin-dependent functions such as cell migration, morphology, and adhesion strengthening [12, 19, 22, 29, 33]. Furthermore, CD151-integrin complexes seemed to be involved in protecting cancer cells in the special case of drugs targeting ErbB2 [25]. Hence, it was unexpected that contributions of CD151 to a more general type of drug resistance would be independent of integrins. Integrin independence is supported by four different types of results. First, drug-sensitizing effects of CD151 ablation were readily observed in nonadherent cells (in which integrins are not engaged). Additional studies using cells on polyHEMA-coated surfaces further indicate that lack of cell-matrix adhesion does not impair drug-sensitizing effects of CD151 ablation. Second, ablation of integrin $\alpha 3$ and $\alpha 6$ subunits did not mimic drug-sensitizing effects of CD151 ablation. Third, the CD151 ${ }^{\mathrm{QRD}}$ mutant functioned comparably to $\mathrm{CD} 151^{\mathrm{WT}}$ in terms of reconstituting drug protection. As seen previously [19], and confirmed in Supplemental Fig. 3b, CD151 ${ }^{\mathrm{QRD}}$ has markedly diminished integrin association properties, due to mutation of a CD151 epitope involved in association with $\alpha 3 \beta 1, \alpha 6 \beta 1, \alpha 6 \beta 4$, and $\alpha 7 \beta 1$ $[12,19]$. Fourth, anti-cancer drugs failed to induce upregulation of integrin-associated CD151. Rather, they selectively induced upregulation of NIA-CD151, and NIA-CD151-QRD 
mutant, consistent with the proposed integrin-independent role of NIA-CD151 in drug resistance. Results were mostly obtained using the epidermoid carcinoma A431 cell line. Results obtained using the other cell lines (e.g., breast carcinoma MDA-MB-231 and lung carcinoma A549; representing other carcinoma types) illustrate the generality of our findings, applicable to cells that either do (A431) or do not (MDA-MB-231, A549) produce excess laminin.

Drug-induced selective upregulation of NIA-CD151 does not diminish the pool of integrin-associated CD151 and does not occur on the cell surface. In addition, upregulation still occurs when using a nonendogenous CMV promoter, and is not seen at the mRNA level, thus pointing to a post-transcriptional regulation mechanism. We suspect that enhanced NIA-CD151 expression results from diminished protein degradation, but this remains to be clarified.

Together, our results emphasize the existence of at least three distinct populations of CD151 (Fig. 5). (a) Integrin-associated CD151 supports adhesion strengthening, migration and invasion and contributes to multiple stages of carcinogenesis [6-10, 34]. In addition, CD151-integrin complexes have been linked to tumor cell sensitivity to agents targeting ErbB2 [25]. (b) By contrast, intracellular NIA-CD151 is selectively upregulated in response to a variety of anti-cancer agents and contributes to drug resistance. (c) The population of NIA-CD151 that appears variably on the cell surface is not upregulated in response to anti-cancer

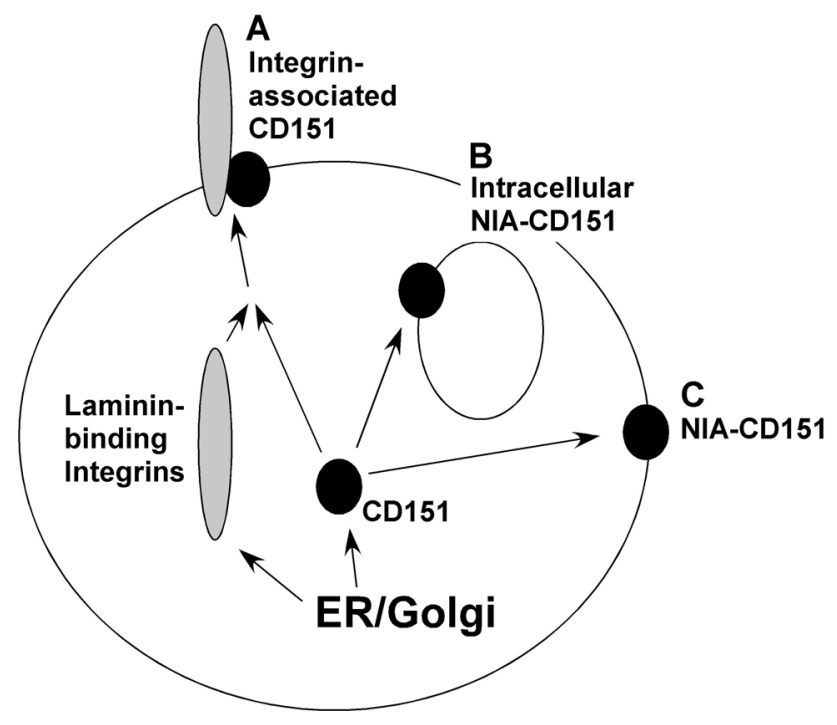

Fig. 5 Schematic diagram of CD151 subpopulations. a During biosynthesis, a subset of CD151 forms complexes with laminin-binding integrins (e.g., $\alpha 3 \beta 1, \alpha 6 \beta 1$, and $\alpha 6 \beta 4$ ), which then appear on the cell surface. b A subset of nonintegrin-associated "NIA"-CD151, localized intracellularly, is upregulated in response to anti-cancer drugs and contributes to drug resistance. c NIA-CD151 also can appear at the cell surface. This population of NIA-CD151 is not upregulated by anti-cancer drugs, but may help to regulate cell migration [10] agents and its functional role is not well defined. However, it was shown that mAb-induced clustering of NIA-CD151 (which is presumably on the cell surface) could inhibit tumor cell metastasis and migration, by a mechanism involving enhanced cell adhesion and perhaps also PKC $\alpha[10,21]$.

NIA-CD151 expression was previously correlated with prostate cancer progression and diminished patient survival [21]. We speculate that this diminished patient survival could at least partly involve elevated NIA-CD151 levels associated with increased drug resistance. CD151 has previously been considered as a cancer target, due to its contributions to multiple stages of carcinogenesis [2]. Our new results, together with prior results linking NIA-CD151 with patient survival [21], now suggest that it may be therapeutically beneficial to selectively target protein epitopes preferentially exposed in NIA-CD151.

How does NIA-CD151 contribute to drug resistance? The conventional PKC isoforms (e.g., PKC $\alpha$ ) can associate with CD151 [6, 28], may be needed for NIA-CD151 regulation of cell migration [21], and can play a role in cancer drug resistance [35]. However, we saw no change in CD151-PKC $\alpha$ association upon drug treatment. Both PKC $\alpha$ [31] and another CD151-associated signaling molecule (PI4K [32]) have been linked to cancer cell survival pathways. However, levels of key intermediates of cancer pro-survival pathways (p-mTOR, p-AKT, and p-ERK) were neither diminished when apoptosis was increased (e.g., in CD151-KO cells), nor elevated when apoptosis was reduced (e.g., in CD151 reconstituted cells). Hence, CD151's role in attenuating anti-cancer drug-induced apoptosis may be independent of activation of pro-survival signaling. Our results suggest that NIA-CD151 can inhibit drug-induced apoptosis, but a more specific mechanism remains to be elucidated.

In conclusion, our novel and unexpected results now focus attention on a previously understudied subpopulation of CD151 that is nonintegrin-associated and appears to be intracellular. By a combination of nonadherent culture conditions, colocalization, correlation, co-immunoprecipitation, integrin ablation, CD151 ablation, CD151 mutation, and reconstitution experiments, CD151 was shown to inhibit drug-induced apoptosis in an integrin-independent manner.

Acknowledgements This work was supported by NIH grant CA42368 (to $\mathrm{MEH}$ ).

OpenAccess This article is distributed under the terms of the Creative Commons Attribution 4.0 International License (http://creativeco mmons.org/licenses/by/4.0/), which permits unrestricted use, distribution, and reproduction in any medium, provided you give appropriate credit to the original author(s) and the source, provide a link to the Creative Commons license, and indicate if changes were made. 


\section{References}

1. Sincock PM, Mayrohofer G, Ashman LK (1997) Localization of the transmembrane 4 superfamily (TM4SF) member PETA-3 (CD151) in normal human tissues - comparison with CD9, CD63, and $\alpha 5 \beta 1$ integrin. J Histochem Cytochem 45:515-525

2. Haeuw JF, Goetsch L, Bailly C, Corvaia N (2011) Tetraspanin CD151 as a target for antibody-based cancer immunotherapy. Biochem Soc Trans 39:553-558

3. Sadej R, Grudowska A, Turczyk L, Kordek R, Romanska HM (2014) CD151 in cancer progression and metastasis: a complex scenario. Lab Invest 94:41-51

4. Zeng P, Wang YH, Si M, Gu JH, Li P, Lu PH, Chen MB (2017) Tetraspanin CD151 as an emerging potential poor prognostic factor across solid tumors: a systematic review and meta-analysis. Oncotarget 8:5592-5602

5. Kumari S, Devi G, Badana A, Dasari VR, Malla RR (2015) CD151A striking marker for cancer therapy. Biomark Cancer 7:7-11

6. Li Q, Yang XH, Xu F, Sharma C, Wang HX, Knoblich K, Rabinovitz I, Granter SR, Hemler ME (2013) Tetraspanin CD151 plays a key role in skin squamous cell carcinoma. Oncogene 32:1772-1783

7. Deng X, Li Q, Hoff J, Novak M, Yang H, Jin H, Erfani SF, Sharma C, Zhou P, Rabinovitz I, Sonnenberg A, Yi A, Zhou P, Stipp CS, Kaetzel DM, Hemler ME, Yang HY (2012) Integrin-associated CD151 drives ErbB2-evoked mammary tumor onset and metastasis. Neoplasia 14:678-689

8. Takeda Y, Kazarov AR, Butterfield CE, Hopkins BD, Benjamin LE, Kaipainen A, Hemler ME (2007) Deletion of tetraspanin Cd151 results in decreased pathologic angiogenesis in vivo and in vitro. Blood 109:1524-1532

9. Testa JE, Brooks PC, Lin JM, Quigley JP (1999) Eukaryotic expression cloning with an antimetastatic monoclonal antibody identifies a tetraspanin (PETA-3/CD151) as an effector of human tumor cell migration and metastasis. Cancer Res 59:3812-3820

10. Zijlstra A, Lewis J, Degryse B, Stuhlmann H, Quigley JP (2008) The inhibition of tumor cell intravasation and subsequent metastasis via regulation of in vivo tumor cell motility by the tetraspanin CD151. Cancer Cell 13:221-234

11. Yauch RL, Kazarov AR, Desai B, Lee RT, Hemler ME (2000) Direct extracellular contact between integrin $\alpha 3 \beta 1$ and TM4SF protein CD151. J Biol Chem 275:9230-9238

12. Sterk LM, Geuijen CA, van Den Berg JG, Claessen N, Weening JJ, Sonnenberg A (2002) Association of the tetraspanin CD151 with the laminin-binding integrins alpha3beta1, alpha6beta1, alpha6beta4 and alpha7beta1 in cells in culture and in vivo. J Cell Sci 115:1161-1173

13. Zevian S, Winterwood NE, Stipp CS (2011) Structure-function analysis of tetraspanin CD151 reveals distinct requirements for tumor cell behaviors mediated by alpha3beta1 versus alpha6beta4 integrin. J Biol Chem 286:7496-7506

14. Sachs N, Kreft M, van den Bergh Weerman MA, Beynon AJ, Peters TA, Weening JJ, Sonnenberg A (2006) Kidney failure in mice lacking the tetraspanin CD151. J Cell Biol 175:33-39

15. Karamatic CV, Burton N, Kagan A, Green CA, Levene C, Flinter F, Brady LR, Daniels G, Anstee DJ (2004) CD151, the first member of the tetraspanin (TM4) superfamily detected on erythrocytes, is essential for the correct assembly of human basement membranes in kidney and skin. Blood 104:2217-2223

16. Vahidnezhad H, Youssefian L, Saeidian AH, Mahmoudi H, Touati A, Abiri M, Kajbafzadeh AM, Aristodemou S, Liu L, McGrath JA, Ertel A, Londin E, Kariminejad A, Zeinali S, Fortina P, Uitto J (2018) Recessive mutation in tetraspanin CD151 causes Kindler syndrome-like epidermolysis bullosa with multi-systemic manifestations including nephropathy. Matrix Biol 66:22-33
17. Kreidberg JA, Donovan MJ, Goldstein SL, Rennke H, Shepherd $\mathrm{K}$, Jones RC, Jaenisch R (1996) $\alpha 3 \beta 1$ integrin has a crucial role in kidney and lung organogenesis. Development 122:3537-3547

18. Margadant C, Charafeddine RA, Sonnenberg A (2010) Unique and redundant functions of integrins in the epidermis. FASEB J 24:4133-4152

19. Kazarov AR, Yang X, Stipp CS, Sehgal B, Hemler ME (2002) An extracellular site on tetraspanin CD151 determines $\alpha 3$ and $\alpha 6$ integrin-dependent cellular morphology. J Cell Biol 158:1299-1309

20. Yamada M, Tamura Y, Sanzen N, Sato-Nishiuchi R, Hasegawa H, Ashman LK, Rubinstein E, Yanez-Mo M, Sanchez-Madrid F, Sekiguchi K (2008) Probing the interaction of tetraspanin CD151 with integrin alpha 3 beta 1 using a panel of monoclonal antibodies with distinct reactivities toward the CD151-integrin alpha 3 beta 1 complex. Biochem J 415:417-427

21. Palmer TD, Martinez CH, Vasquez C, Hebron KE, Jones-Paris C, Arnold SA, Chan SM, Chalasani V, Gomez-Lemus JA, Williams AK, Chin JL, Giannico GA, Ketova T, Lewis JD, Zijlstra A (2014) Integrin-free tetraspanin CD151 can inhibit tumor cell motility upon clustering and is a clinical indicator of prostate cancer progression. Cancer Res 74:173-187

22. Serru V, Naour FL, Billard M, Azorsa DO, Lanza F, Boucheix C, Rubinstein E (1999) Selective tetraspan-integrin complexes (CD81/alpha4beta1, CD151/alpha3beta1, CD151/alpha6beta1) under conditions disrupting tetraspan interactions. Biochem $\mathbf{J}$ 340(Pt 1):103-111

23. Holohan C, Van SS, Longley DB, Johnston PG (2013) Cancer drug resistance: an evolving paradigm. Nat Rev Cancer 13:714-726

24. Damiano JS (2002) Integrins as novel drug targets for overcoming innate drug resistance. Curr Cancer Drug Targets 2:37-43

25. Yang XH, Flores LM, Li Q, Zhou P, Xu F, Krop IE, Hemler ME (2010) Disruption of laminin-integrin-CD151-focal adhesion kinase axis sensitizes breast cancer cells to ErbB2 antagonists. Cancer Res 70:2256-2263

26. Ran FA, Hsu PD, Wright J, Agarwala V, Scott DA, Zhang F (2013) Genome engineering using the CRISPR-Cas9 system. Nat Protoc 8:2281-2308

27. Cheong TC, Compagno M, Chiarle R (2016) Editing of mouse and human immunoglobulin genes by CRISPR-Cas9 system. Nat. Commun. 7:10934

28. Zhang XA, Bontrager AL, Hemler ME (2001) TM4SF proteins associate with activated $\mathrm{PKC}$ and Link PKC to specific beta1 integrins. J Biol Chem 276:25005-25013

29. Lammerding J, Kazarov AR, Huang H, Lee RT, Hemler ME (2003) Tetraspanin CD151 regulates alpha6beta1 integrin adhesion strengthening. Proc Natl Acad Sci USA 100:7616-7621

30. Yauch RL, Berditchevski F, Harler MB, Reichner J, Hemler ME (1998) Highly stoichiometric, stable and specific association of integrin $\alpha 3 \beta 1$ with CD151 provides a major link to phosphatidylinositol 4-kinase and may regulate cell migration. Mol Biol Cell 9:2751-2765

31. Schonwasser DC, Marais RM, Marshall CJ, Parker PJ (1998) Activation of the mitogen-activated protein kinase/extracellular signalregulated kinase pathway by conventional, novel, and atypical protein kinase C isotypes. Mol Cell Biol 18:790-798

32. Chu KM, Minogue S, Hsuan JJ, Waugh MG (2010) Differential effects of the phosphatidylinositol 4-kinases, PI4KIIalpha and PI4KIIIbeta, on Akt activation and apoptosis. Cell Death Dis 1:e106

33. Sincock PM, Fitter S, Parton RG, Berndt MC, Gamble JR, Ashman LK (1999) PETA-3/CD151, a member of the transmembrane 4 superfamily, is localised to the plasma membrane and endocytic system of endothelial cells, associates with multiple integrins and modulates cell function. J Cell Sci 112:833-844

34. Varzavand A, Drake JM, Svensson RU, Herndon ME, Zhou B, Henry MD, Stipp CS (2013) Integrin alpha3beta1 regulates 
tumor cell responses to stromal cells and can function to suppress prostate cancer metastatic colonization. Clin Exp Metastasis 30:541-552

35. Gravitt KR, Ward NE, Fan D, Skibber JM, Levin B, O'Brian CA (1994) Evidence that protein kinase C-alpha activation is a critical event in phorbol ester-induced multiple drug resistance in human colon cancer cells. Biochem Pharmacol 48:375-381
Publisher's Note Springer Nature remains neutral with regard to jurisdictional claims in published maps and institutional affiliations. 